Supporting Information

\title{
Microwave-assisted Synthesis of Zirconium
}

\section{Phosphate Nanoplatelets Supported Ru-Anadem}

\section{Nano-structures and Their Catalytic Study for the Hydrogenation of Acetophenone}

Xiaoyu Li,${ }^{\dagger}$ Guodong Ding,${ }^{\star}$ Brena, L. Thompson,${ }^{\star}$ Leiduan Hao,${ }^{\star}$ Derek A. Deming,${ }^{\star}$ Zachariah M. Heiden, ${ }^{\ddagger}$ and Qiang Zhang*,†,

${ }^{\dagger}$ Materials Science and Engineering Program, Washington State University, Pullman, WA 99163, USA

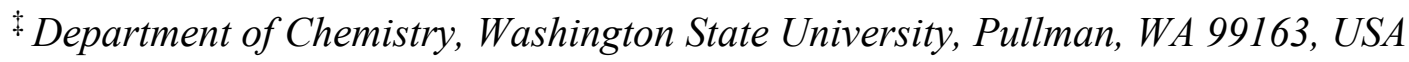

Email:q.zhang@wsu.edu 
Table S1. Summary of catalytic applications with metal-ZrP materials.

\begin{tabular}{|c|c|c|c|}
\hline NO. & Materials & Application & Ref. \\
\hline 1 & $\begin{array}{c}\mathrm{Rh}(\mathrm{III}) \text { and } \mathrm{Ir}(\mathrm{III}) \\
\text { complexes intercalated } \\
\text { into } \mathrm{ZrP}\end{array}$ & Visible light-driven $\mathrm{H}_{2}$ production & 1 \\
\hline 2 & $\begin{array}{c}\text { Co-Ru immobilized on } \\
\mathrm{ZrP} / \mathrm{SiO}_{2}\end{array}$ & Fisher-Tropsch reaction & 2 \\
\hline 3 & $\begin{array}{l}\text { Pd nanoparticles supported } \\
\text { on ZrP }\end{array}$ & Heck reaction & 3 \\
\hline 4 & Ag@AgCl/nanosized ZrP & Degradation of methylene blue & 4 \\
\hline 5 & $\mathrm{Ru} / \mathrm{SiO}_{2}-\mathrm{ZrP}$ & Conversion of glycerol to 1-propanol & 5 \\
\hline 6 & Ti-ZrP & $\begin{array}{l}\text { Enhanced photodegradation ability for methylene } \\
\text { blue }\end{array}$ & 6 \\
\hline 7 & $\mathrm{Cu}-\mathrm{ZrP}$ & Selective oxidation of alcohols & 7 \\
\hline 8 & $\mathrm{Cr}-\mathrm{ZrP}$ & $\begin{array}{c}\text { Catalyzing dehydration of carbohydrates to produce } \\
\text { biofuel precursor }\end{array}$ & 8 \\
\hline 9 & $\mathrm{Pt}, \mathrm{Pd} / \mathrm{ZrP}$ & Hydrodeoxygenation reaction of dibenzofuran & 9 \\
\hline 10 & $\begin{array}{l}\mathrm{Ag} \text { nanoclusters } \\
\text { intercalated in } \mathrm{ZrP}\end{array}$ & Hydrogenation of nitroaromatics & 10 \\
\hline 11 & CoZrP/KIT-6 & CO hydrogenation to hydrocarbons & 11 \\
\hline 12 & $\mathrm{Fe}, \mathrm{Mn}-\mathrm{ZrP}$ & Oxidation of cyclohexane & 12 \\
\hline 13 & $\mathrm{Au} / \mathrm{ZrP}$ composites & Catalysis for 4-nitrophenol reduction & 13 \\
\hline 14 & $\mathrm{Zn}-\mathrm{ZrP}$ & In situ polymerization of polylactide nanocomposites & 14 \\
\hline 15 & $\mathrm{Ni} / \mathrm{ZrP}$ & Selective depolymerization of lignin & 15 \\
\hline 16 & $\begin{array}{l}\text { Transition metal-modified } \\
\text { ZrP }\end{array}$ & Electrocatalysts for oxygen evolution reaction & 16 \\
\hline
\end{tabular}



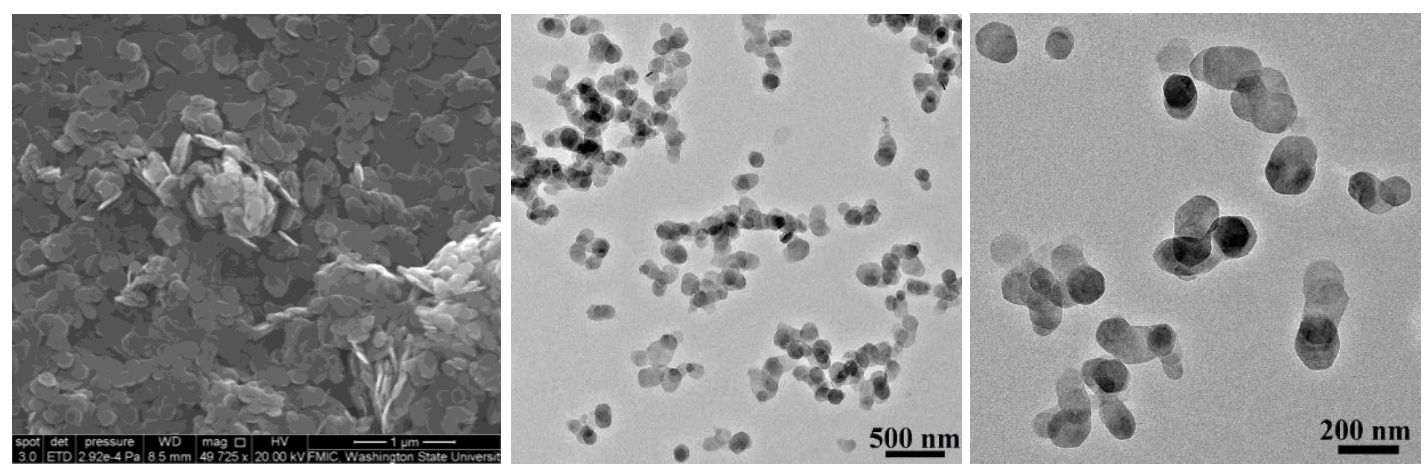

Figure S1. SEM and TEM images of $\alpha-Z r P$ nanoplatelets. 


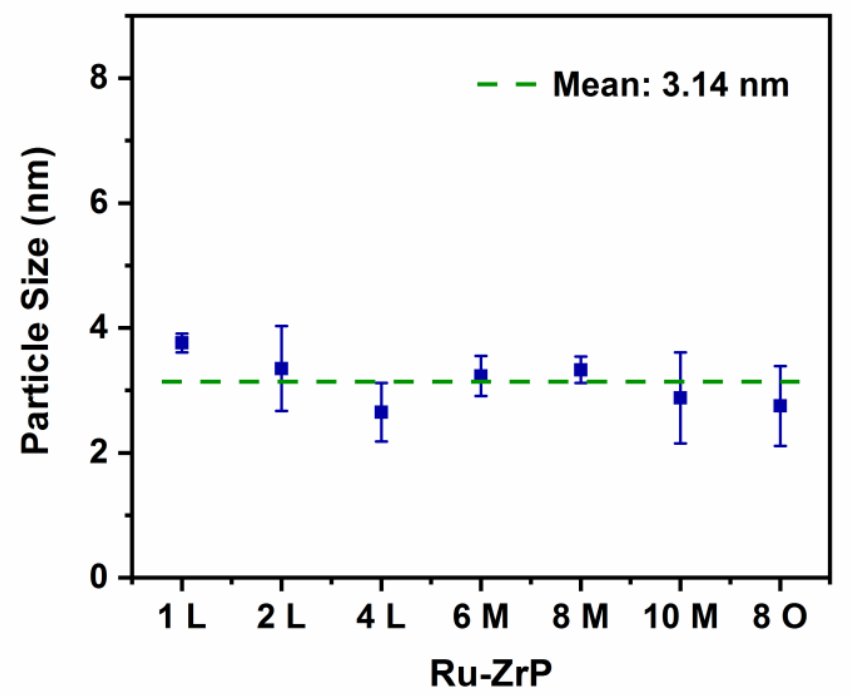

Figure S2. Average sizes (error bar: standard deviation) of Ru NPs at different synthesis conditions. 


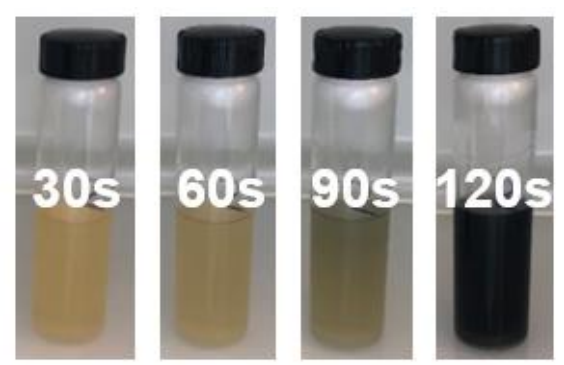

Figure S3. Color change during the microwave synthesis of Ru-ZrP. 


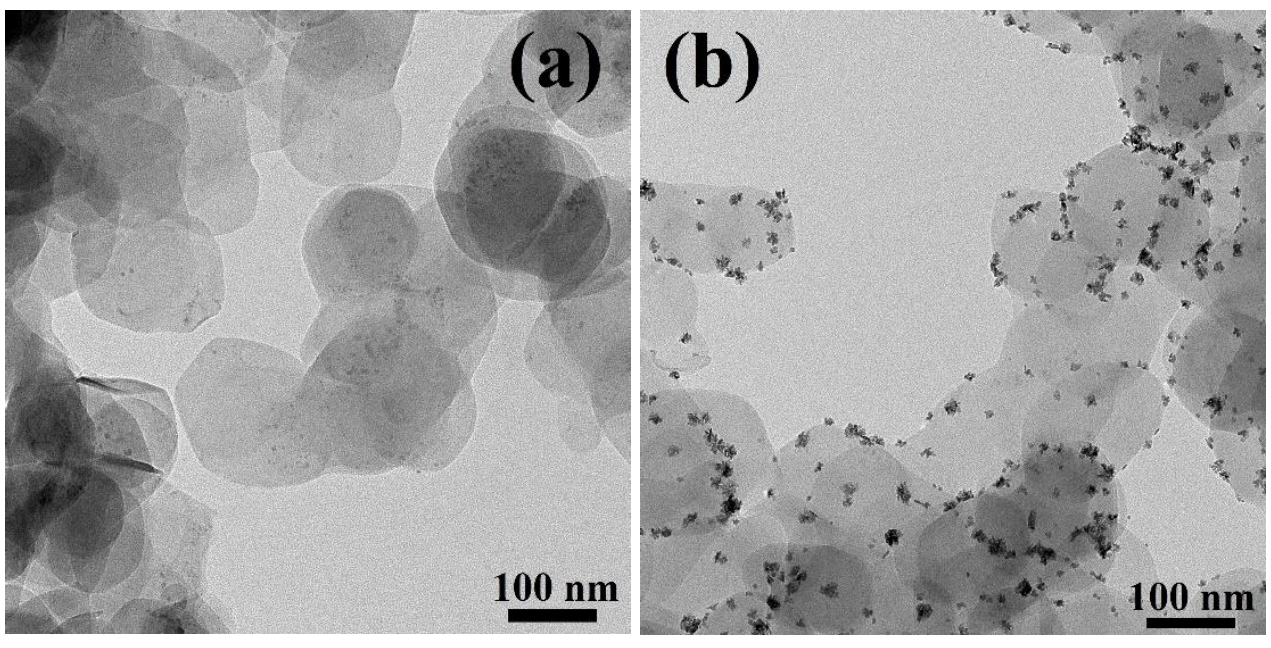

Figure S4. TEM images of Ru-ZrP-2L prepared at different reaction time. a) 1 min and b) 1.5 min with low power microwave. 

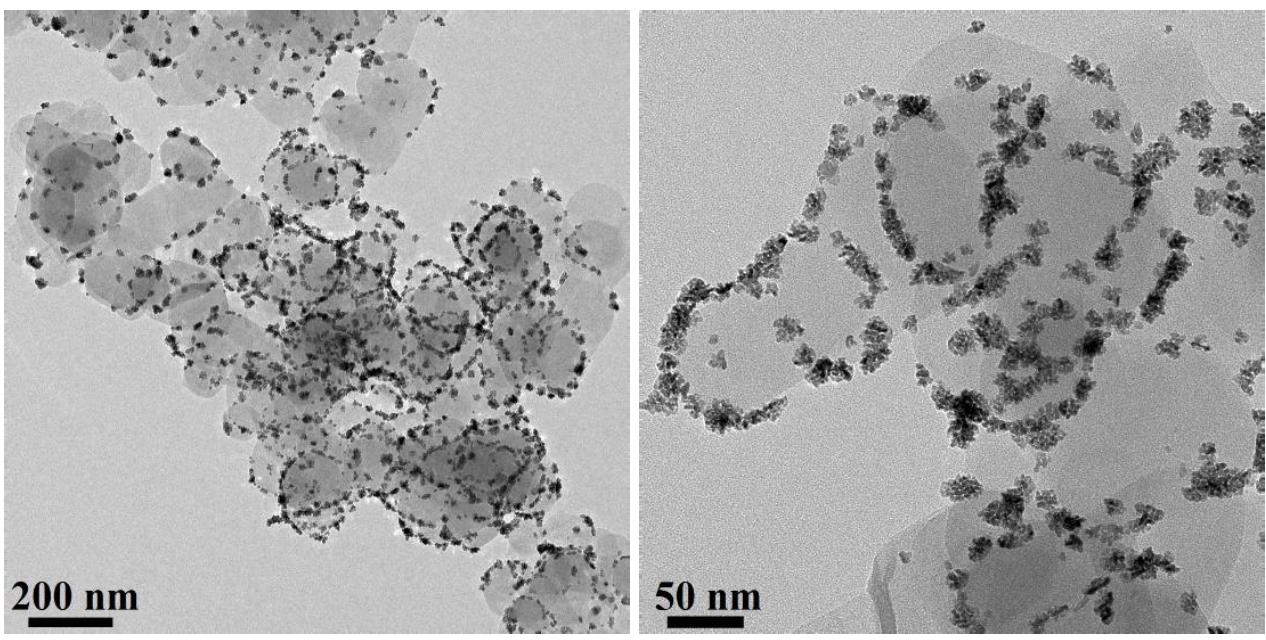

Figure S5. TEM images of Ru-ZrP-8O, prepared with an oil bath at $177^{\circ} \mathrm{C}$. 
Table S2. $\mathrm{N}_{2}$ adsorption properties and elemental analysis for $\mathrm{Ru}-\mathrm{ZrP}$ catalysts and $\alpha-\mathrm{ZrP}$ nanoplatelets.

\begin{tabular}{cccc}
\hline Sample & $\mathbf{S}_{\text {Beт }}\left(\mathbf{m}^{\mathbf{2}} \mathbf{g}^{-\mathbf{1}}\right)$ & $\mathbf{V}_{\text {total }}\left(\mathbf{c m}^{\mathbf{3}} \mathbf{g}^{-1}\right)$ & $\mathbf{R u} / \mathbf{Z r}(\mathbf{\%})^{\boldsymbol{a}}$ \\
\hline$\alpha-Z r P$ & 49.08 & 0.11 & $/$ \\
Ru-ZrP-4 L & 45.03 & 0.09 & 2.38 \\
Ru-ZrP-6 M & 47.54 & 0.10 & 5.97 \\
Ru-ZrP-8 M & 50.50 & 0.12 & 6.48 \\
Ru-ZrP-10 M & 49.34 & 0.11 & 7.22 \\
Ru@ZrP & 51.58 & 0.11 & 0.35 \\
Ru-ZrP-8 O & 53.64 & 0.10 & 5.14 \\
\hline
\end{tabular}

${ }^{a}$ Mass ratio between $\mathrm{Ru}$ and $\mathrm{Zr}$ was determined by ICP-OES. 
(a)

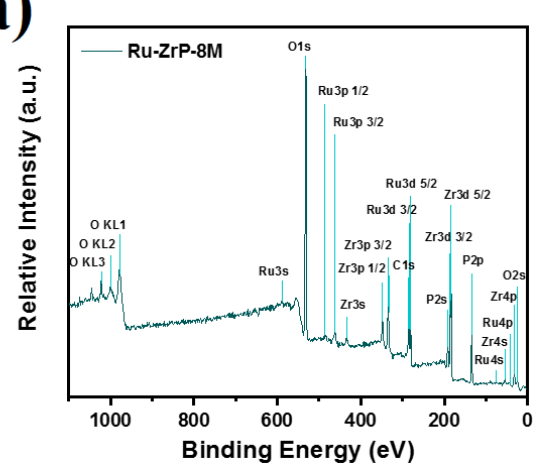

(c)

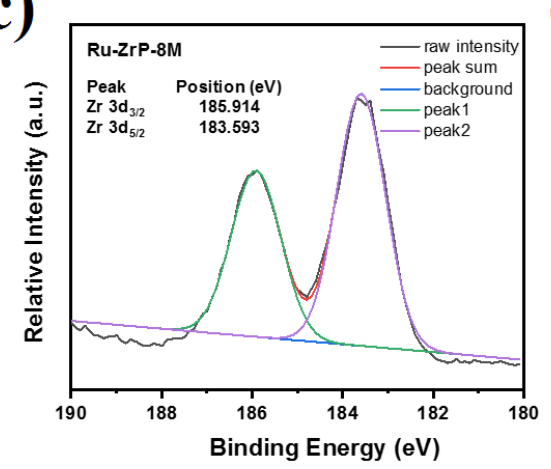

(b)

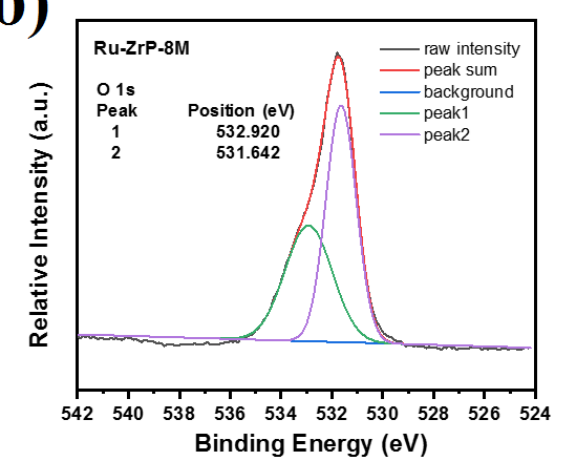

(d)

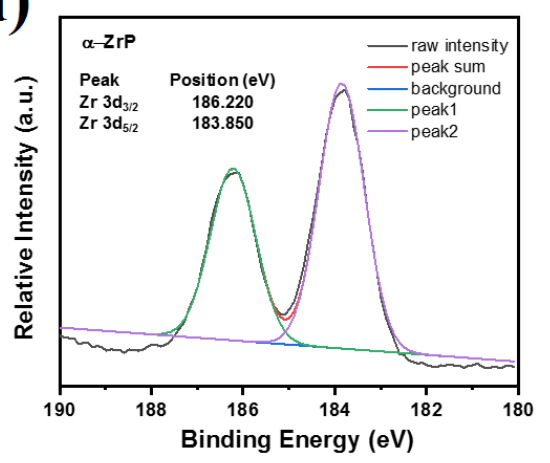

Figure S6. XPS spectra of (a) wide region, (b) O 1s, (c) Zr 3d of Ru-ZrP-8M and (d) Zr 3d of $\alpha-\mathrm{ZrP}$. 

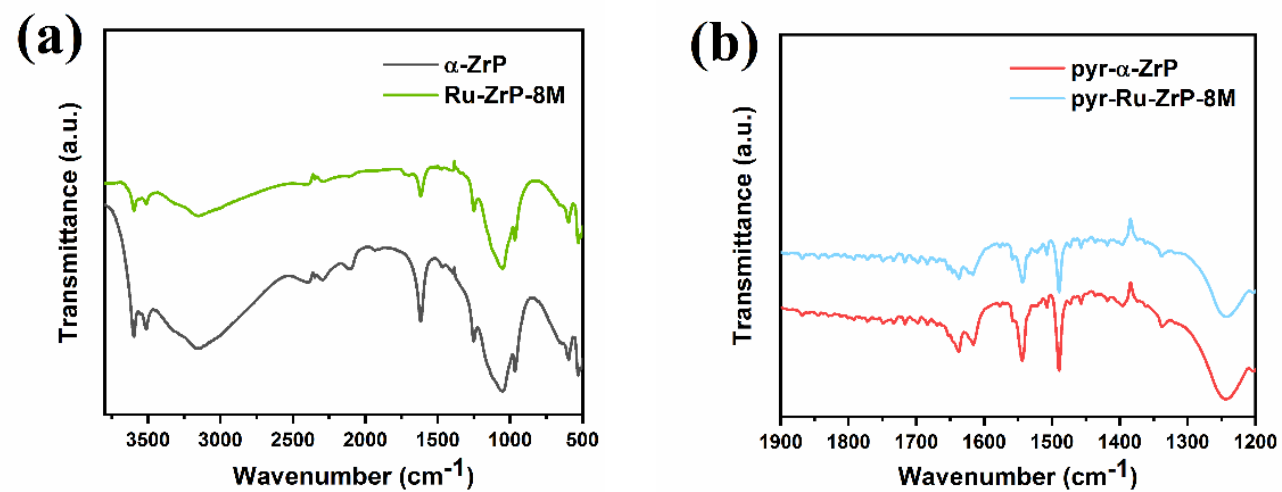

Figure S7. FT-IR spectra of $\alpha-\mathrm{ZrP}$ and Ru-ZrP-8M comparison (a), and their pyridine desorbed samples (b). 


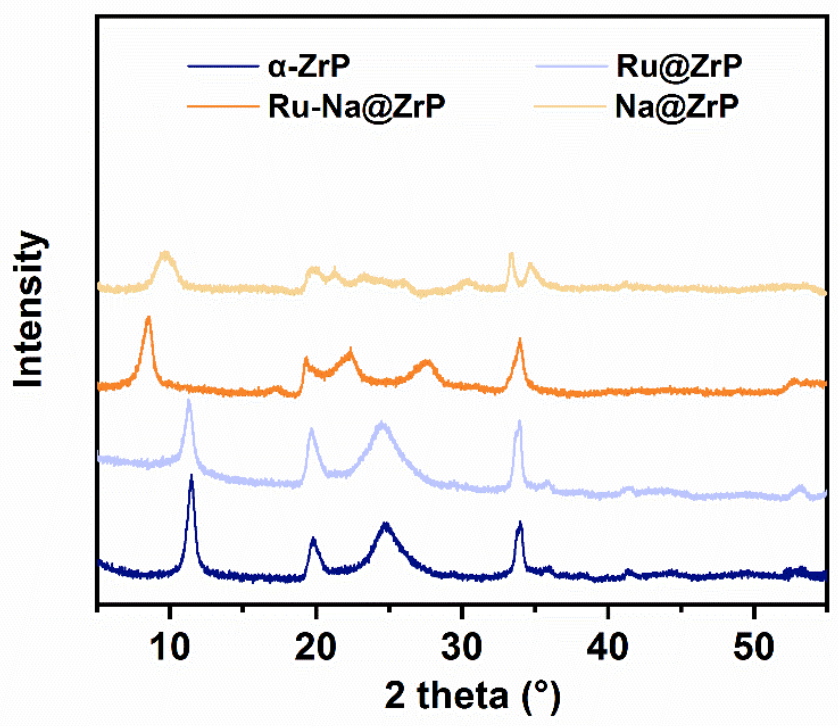

Figure S8. PXRD patterns of $\alpha-\mathrm{ZrP}, \mathrm{Ru} @ \mathrm{ZrP}, \mathrm{Na} @ \mathrm{ZrP}$, and Ru-Na@ZrP nanoplatelets. 


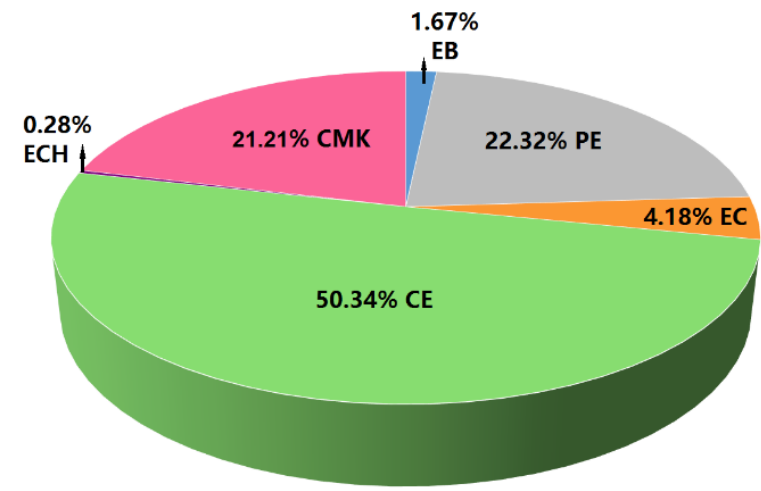

Figure S9. Products' yields distribution for Ru-ZrP-6M catalyst (Entry 2, Table 2). 
Table S3. Comparison of catalytic efficiency by using metal catalysts for acetophenone hydrogenation.

\begin{tabular}{|c|c|c|c|c|c|}
\hline Materials & $\begin{array}{l}\text { Reaction } \\
\text { Conditions }\end{array}$ & Yields of Products & Time & $\mathrm{H}_{2}$ pressure & Refs. \\
\hline $\mathrm{Ru}-\mathrm{ZrP}$ & $23^{\circ} \mathrm{C}$ & 95\% 1-cyclohexylethanol & $120 \mathrm{~min}$ & 40 bar (4 MPa) & $\begin{array}{l}\text { This } \\
\text { work }\end{array}$ \\
\hline $\mathrm{Pt} / \mathrm{Al}_{2} \mathrm{O}_{3}{ }^{a}$ & $25^{\circ} \mathrm{C}$ & $\begin{array}{c}\text { 71.6\% 1-phenylethanol, } \\
21.9 \% \text { cyclohexylmethylketone, } \\
6.5 \% \text { 1-cyclohexylethanol }\end{array}$ & $60 \mathrm{~min}$ & $1 \mathrm{bar}(0.1 \mathrm{MPa})$ & 17 \\
\hline $\begin{array}{c}\text { Pt-Fe } \\
\text { nanowires }\end{array}$ & $70^{\circ} \mathrm{C}$ & 91.7\% 1-phenylethanol & $200 \mathrm{~min}$ & $1 \mathrm{bar}(0.1 \mathrm{MPa})$ & 18 \\
\hline $\mathrm{Rh} / \mathrm{C}, \mathrm{Rh} / \mathrm{Al}_{2} \mathrm{O}_{3}$ & $80^{\circ} \mathrm{C}$ & 56-88\% 1-phenylethanol & $60 \mathrm{~min}$ & $20 \operatorname{bar}(2 \mathrm{MPa})$ & 19 \\
\hline $\mathrm{Ni} / \mathrm{SiO}_{2}$ & $80-100^{\circ} \mathrm{C}$ & $92 \%$ 1-phenylethanol & $120 \mathrm{~min}$ & 10 bar (1 MPa) & 20 \\
\hline $\mathrm{RuB} / \mathrm{SiO}_{2}{ }^{b}$ & $60^{\circ} \mathrm{C}$ & $\begin{array}{c}22 \% 1 \text {-phenylethanol, } \\
36 \% \text { cyclohexylmethylketone, } \\
42 \% 1 \text {-cyclohexylethanol }\end{array}$ & $60 \min$ & $9 \operatorname{bar}(0.9 \mathrm{MPa})$ & 21 \\
\hline $\mathrm{Ru} / \mathrm{AC}-\mathrm{SO}_{3} \mathrm{H}^{c}$ & $150^{\circ} \mathrm{C}$ & $\begin{array}{l}32 \% \text { ethylbenzene, } \\
66 \% \text { ethylcyclohexane, } \\
2 \% \text { 1-phenylethanol }\end{array}$ & $180 \mathrm{~min}$ & 40 bar (4 MPa) & 22 \\
\hline
\end{tabular}

${ }^{a}$ Total conversion of acetophenone is $61 \%$.

${ }^{b}$ Total conversion of acetophenone is $90 \%$.

${ }^{c}$ Sulfonated active carbon $\left(\mathrm{AC}-\mathrm{SO}_{3} \mathrm{H}\right)$ with a strong acid center favors deoxygenation. 


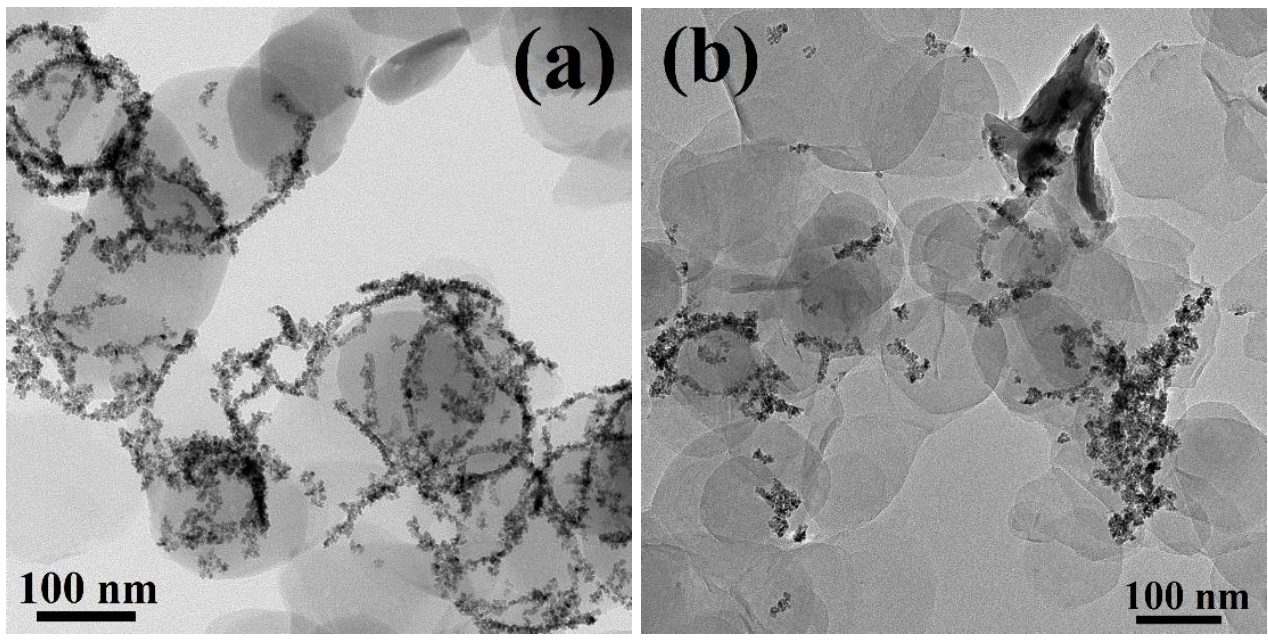

Figure S10. TEM images for Ru-ZrP-8M catalyst after running $18 \mathrm{~h}$ at $23^{\circ} \mathrm{C}(\mathrm{a})$ and $2 \mathrm{~h}$ at $120^{\circ} \mathrm{C}(\mathrm{b})$. 


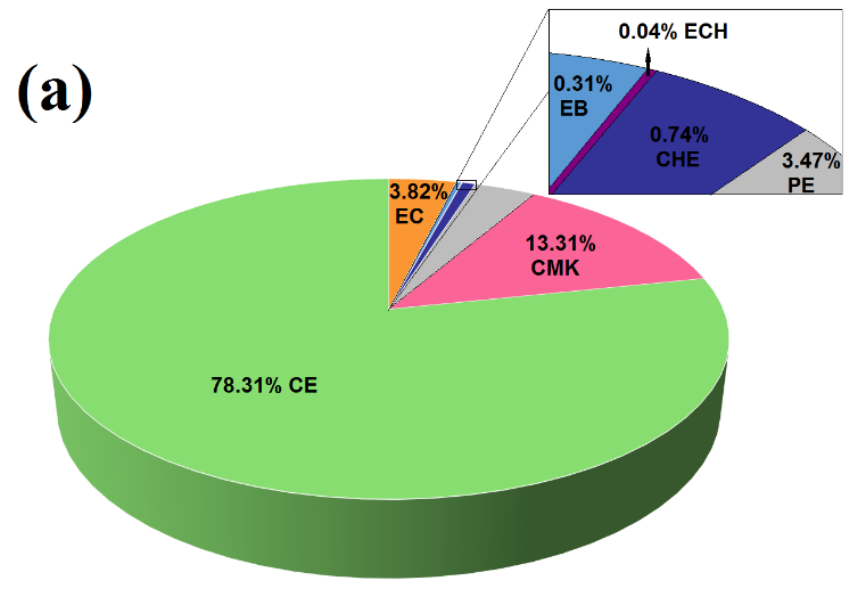

(b)

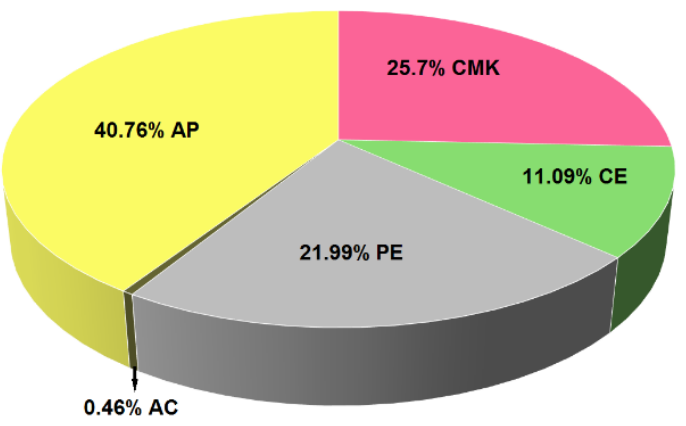

Figure S11. Products and residues distribution for Ru-ZrP-8M catalyst work at different pressure of hydrogen: (a) $2 \mathrm{MPa}$ and (b) $1 \mathrm{MPa}$. All the other reaction conditions are the same. 


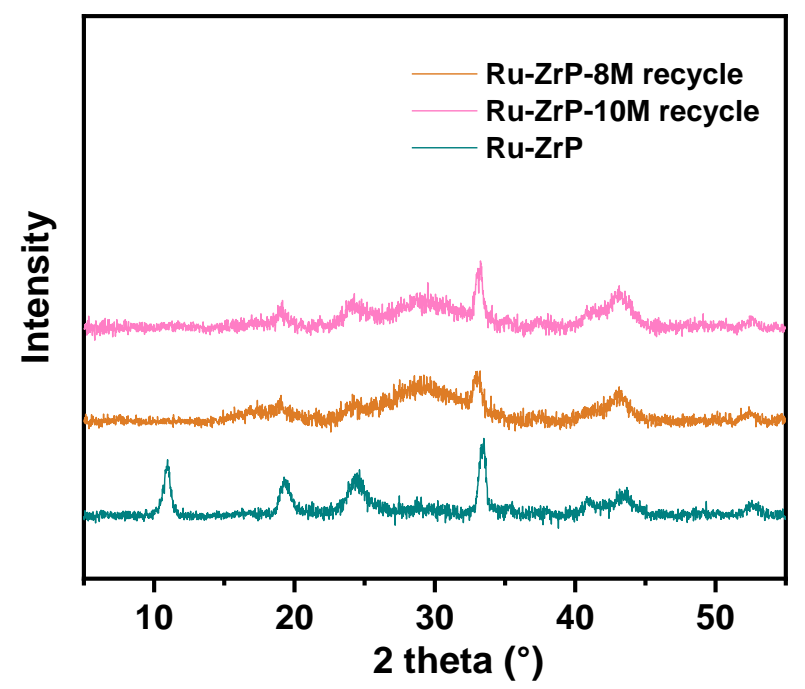

Figure S12. Comparison of PXRD patterns of recycling catalysts. Recycled Ru-ZrP-8M, Ru-ZrP-10M, which have been used for room temperature reaction, and fresh-made Ru-ZrP. 

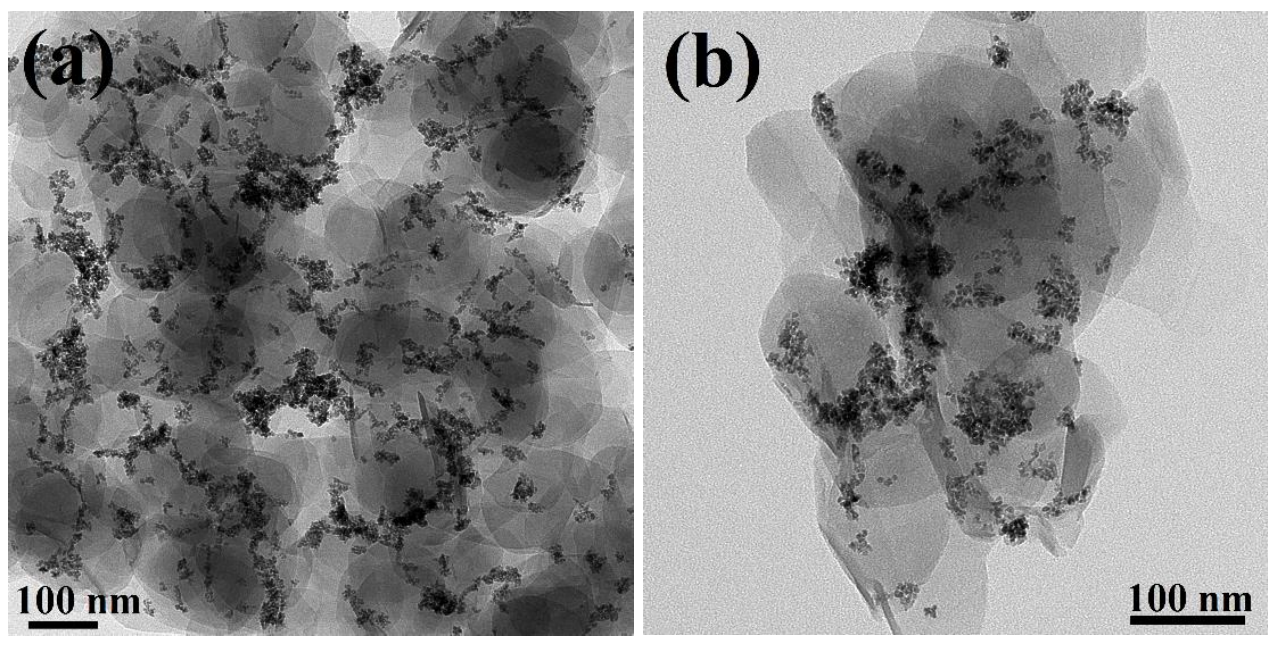

Figure S13. TEM images for recycling catalyst Ru-ZrP-8M. Entry $3\left(23^{\circ} \mathrm{C}, 2 \mathrm{~h}\right)$ at $4^{\text {th }}$ run. (a) and (b) represent different areas. 


\section{References}

(1) Mori, K.; Aoyama, J.; Kawashima, M.; Yamashita, H. Visible-light driven $\mathrm{H}_{2}$ production utilizing iridium and rhodium complexes intercalated into a zirconium phosphate layered matrix. Dalton Trans. 2014, 43 (27), 10541-10547.

(2) Bae, J. W.; Park, S. J.; Woo, M. H.; Cheon, J. Y.; Ha, K. S.; Jun, K. W.; Lee, D. H.; Jung, H. M. Enhanced Catalytic Performance by Zirconium Phosphate-Modified $\mathrm{SiO}_{2}$-Supported RuCo Catalyst for Fischer-Tropsch Synthesis. ChemCatChem 2011, 3 (8), 1342-1347.

(3) Petrucci, C.; Cappelletti, M.; Piermatti, O.; Nocchetti, M.; Pica, M.; Pizzo, F.; Vaccaro, L. Immobilized palladium nanoparticles on potassium zirconium phosphate as an efficient recoverable heterogeneous catalyst for a clean Heck reaction in flow. J. Mol. Catal. A: Chem. 2015, 401, 27-34.

(4) Pica, M.; Nocchetti, M.; Ridolfi, B.; Donnadio, A.; Costantino, F.; Gentili, P. L.; Casciola, M. Nanosized zirconium phosphate/ $\mathrm{AgCl}$ composite materials: a new synergy for efficient photocatalytic degradation of organic dye pollutants. J. Mater. Chem. A 2015, 3 (10), 5525-5534.

(5) Wang, M.; Yang, H.; Xie, Y.; Wu, X.; Chen, C.; Ma, W.; Dong, Q.; Hou, Z. Catalytic transformation of glycerol to 1-propanol by combining zirconium phosphate and supported Ru catalysts. RSC Adv. 2016, 6 (35), 29769-29778.

(6) Zhou, Y.; Wang, A.; Wang, Z.; Chen, M.; Wang, W.; Sun, L.; Liu, X. Titanium functionalized $\alpha-$ zirconium phosphate single layer nanosheets for photocatalyst applications. $R S C A d v$. 2015, 5 (114), 9396993978.

(7) Hajipour, A. R.; Karimi, H. Selective oxidation of alcohols over copper zirconium phosphate. Chinese J. Catal. 2014, 35 (9), 1529-1533.

(8) Liu, B.; Ba, C.; Jin, M.; Zhang, Z. Effective conversion of carbohydrates into biofuel precursor 5hydroxymethylfurfural (HMF) over Cr-incorporated mesoporous zirconium phosphate. Ind. Crops Prod. 2015, 76, 781-786.

(9) Ballesteros-Plata, D.; Infantes-Molina, A.; Rodríguez-Aguado, E.; Braos-García, P.; RodríguezCastellón, E. Lamellar zirconium phosphates to host metals for catalytic purposes. Dalton Trans. 2018, 47 (9), 3047-3058.

(10) Gong, H.; Lin, L.; Zhao, X.; Li, H.; Li, D.; Xu, Z.; Chen, M.; Huang, R.; Hou, Z. Atomically precise $\mathrm{Ag}$ nanoclusters intercalated in zirconium pyrophosphate for efficient hydrogenation of nitroaromatics. Appl. Catal., A 2019, 574, 1-9.

(11) Cho, J. M.; Kasipandi, S.; Park, Y. M.; Bae, J. W. Spatially confined cobalt nanoparticles on zirconium phosphate-modified KIT-6 for an enhanced stability of CO hydrogenation to hydrocarbons. Fuel 2019, 239, 547-558.

(12) Khare, S.; Shrivastava, P.; Chokhare, R.; Kirar, J. S.; Parashar, S. $\alpha$-Zirconium phosphate supported metal-salen complex: synthesis, characterization and catalytic activity for cyclohexane oxidation. J. Porous Mater. 2017, 24 (4), 855-866.

(13) Lai, G.-H.; Huang, T.-C.; Pai, Y.-H.; Huang, B.-S.; Tsai, M.-H.; Yang, T.-I.; Chung, Y.-H. Preparation of highly-stable and recyclable novel Au/ZrP composite catalyst for 4-nitrophenol reduction. J.Taiwan Inst. Chem. E. 2019, 95, 525-531.

(14) Xu, Y.; Lin, L.; Zeng, S.; Liu, J.; Xiao, M.; Wang, S.; Meng, Y.; Sun, L. Synthesis of Polylactide Nanocomposites Using $\alpha$-Zirconium Phosphate Nanosheets Supported Zinc Catalyst via in situ Polymerization. ACS Appl. Polym. Mater. 2019, 1, 1382-1389..

(15) Ma, H.; Li, H.; Zhao, W.; Li, L.; Liu, S.; Long, J.; Li, X. Selective depolymerization of lignin catalyzed by nickel supported on zirconium phosphate. Green Chem. 2019, 21 (3), 658-668.

(16) Sanchez, J.; Ramos-Garcés, M. V.; Narkeviciute, I.; Colón, J. L.; Jaramillo, T. F. Transition metalmodified zirconium phosphate electrocatalysts for the oxygen evolution reaction. Catalysts 2017, 7(5), 132. 
(17) Chen, M.; Maeda, N.; Baiker, A.; Huang, J. Molecular insight into Pt-catalyzed chemoselective hydrogenation of an aromatic ketone by in situ modulation-excitation IR spectroscopy. ACS Catal. 2012, 2 (9), 2007-2013.

(18) Wu, W.; Li, J.; Chen, Z.; Chen, W.; Pang, H.; Ma, K.; Zeng, J. High-index facets of PtFe nanowires induce steric effect on selective hydrogenation of acetophenone. J. Catal. 2019, 373, 209-214.

(19) Yoshida, H.; Onodera, Y.; Fujita, S.-i.; Kawamori, H.; Arai, M. Solvent effects in heterogeneous selective hydrogenation of acetophenone: differences between $\mathrm{Rh} / \mathrm{C}$ and $\mathrm{Rh} / \mathrm{Al}_{2} \mathrm{O}_{3}$ catalysts and the superiority of water as a functional solvent. Green Chem. 2015, 17 (3), 1877-1883.

(20) Bertero, N. M.; Trasarti, A. F.; Apesteguía, C. R.; Marchi, A. J. Solvent effect in the liquid-phase hydrogenation of acetophenone over $\mathrm{Ni} / \mathrm{SiO}_{2}$ : A comprehensive study of the phenomenon. Appl. Catal., A 2011, 394 (1-2), 228-238.

(21) Casagrande, M.; Storaro, L.; Talon, A.; Lenarda, M.; Frattini, R.; Rodrıguez-Castellón, E.; MairelesTorres, P. Liquid phase acetophenone hydrogenation on $\mathrm{Ru} / \mathrm{Cr} / \mathrm{B}$ catalysts supported on silica. J. Mol. Catal. A: Chem. 2002, 188 (1-2), 133-139.

(22) Wang, J.; Zhang, Y.; Zhang, M.; Wang, Z.; Zhang, M. Hydrodeoxygenation of acetophenone over a sulfonated carbon-supported ruthenium catalyst. Catal. Today 2018, 314, 164-169. 